\title{
Транспортные свойства гетероэпитаксиальных пленок на основе теллурида висмута в сильных магнитных полях
}

\author{
(С) Л.Н. Лукьянова, Ю.А. Бойков, О.А. Усов, В.А. Данилов, М.П. Волков \\ Физико-технический институт им. А.Ф. Иофрфе Российской академии наук, \\ 194021 Санкт-Петербург, Россия \\ E-mail: lidia.lukyanova@mail.ioffe.ru
}

(Получена 27 декабря 2016 г. Принята к печати 12 января 2017 г.)

\begin{abstract}
Проведены исследования температурных и магнитополевых зависимостей гальваномагнитных свойств гетероэпитаксиальных пленок $n-\mathrm{Bi}_{2} \mathrm{Te}_{3}$ в магнитных полях до 14 Тл при низких температурах. Показано, что ступени на магнитополевых зависимостях квантового эффекта Холла и плато на температурных зависимостях магнетосопротивления в пленках обусловлены топологическими поверхностными состояниями фермионов Дирака.
\end{abstract}

DOI: $10.21883 /$ FTP.2017.07.44632.18

\section{1. Введение}

Термоэлектрические материалы на основе теллурида висмута в настоящее время приобрели широкую известность как эффективные топологические изоляторы. В этих материалах благодаря сильному спин-орбитальному взаимодействию электронов в поверхностном слое возникает металлическая проводимость с линейной дисперсией и специфической спиновой текстурой, характерной для дираковских фермионов, а объем является изолятором $[1,2]$. Аномальные свойства поверхностных электронных состояний топологических изоляторов в настоящее время используются в оптоэлектронике $[3,4]$, особое внимание уделяется применению топологических поверхностных состояний в термоэлектричестве [5]. Рассматривается возможность повышения термоэлектрической эффективности за счет увеличения подвижности носителей заряда вследствие явления сверхтекучести при конденсации топологических экситонов на поверхности гетероструктурированных пленок на основе халькогенидов висмута и сурьмы [6].

В работе проведено исследование температурных и магнитополевых зависимостей гальваномагнитных свойств в гетероэпитаксиальных пленках $n-\mathrm{Bi}_{2} \mathrm{Te}_{3}$ в сильных магнитных полях до 14 Тл. Квантовые осцилляции магнетосопротивления, квантовый эффект Холла в области низких температур и особенности температурных зависимостей этих эффектов при топологическом фазовом переходе металл-изолятор в магнитном поле используются для анализа свойств топологических поверхностных состояний в халькогенидных термоэлектриках.

\section{2. Квантовый эффект Холла и осцилляции магнетосопротивления}

В гетероэпитаксиальных пленках $n-\mathrm{Bi}_{2} \mathrm{Te}_{3}$ были исследованы зависимости магнетосопротивления $R_{x x}$ и холловского сопротивления $R_{H}$ от магнитного поля и температуры в интервале $4.2-100 \mathrm{~K}$ в магнитных полях до 14 Тл. Для анализа величин $R_{H}$ и $R_{x x}$ от магнитного поля $B$ из экспериментальных зависимостей $R_{H}(B)$ и $R_{x x}(B)$ был удален фон, аппроксимированный полиномами 3-й степени (рис. 1, кривые 1,3 ) и на зависимостях $R_{H}(B)$ при $T=4.2 \mathrm{~K}$ были обнаружены ступени, характерные для квантового эффекта Холла (рис. 1, кривая 4), соответствующие осцилляциям магнетосопротивления на зависимости $R_{x x}(B)$ (рис. 1 , кривая 2) в интервале магнитных полей 5-14 Тл.

Такие ступени типичны для трехмерных топологических изоляторов, к которым относятся пленки на основе

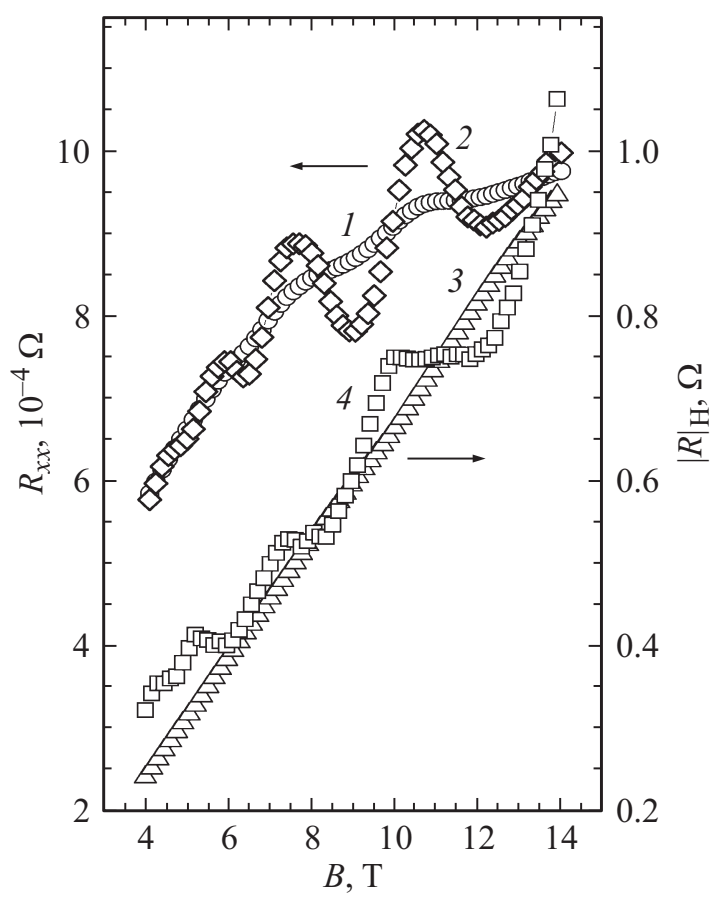

Рис. 1. Зависимости магнетосопротивления $R_{x x}(1)$, холловского сопротивления $R_{H}(3)$, осцилляций магнетосопротивления $(2)$ и холловского сопротивления (4) от магнитного поля $B$ в пленке $n-\mathrm{Bi}_{2} \mathrm{Te}_{3}$. 


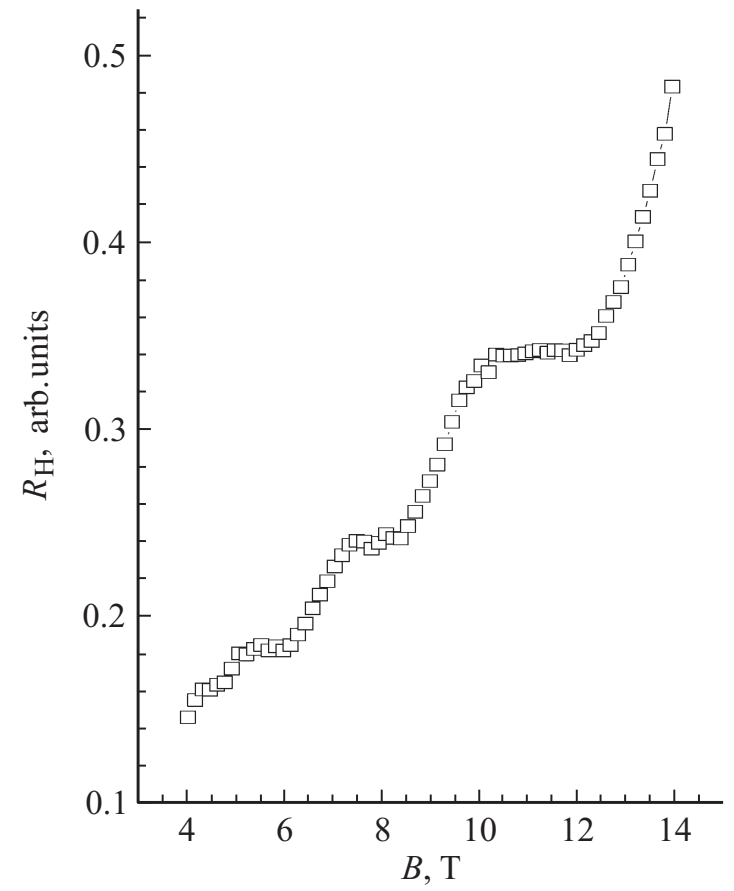

Рис. 2. Зависимость холловского сопротивления $R_{H}$ от магнитного поля $B$ в пленке $n-\mathrm{Bi}_{2} \mathrm{Te}_{3}$ после вычитания фонового сигнала по отношению к одному квинтету.

халькогенидов висмута и сурьмы [7]. В отличие от целочисленного квантового эффекта Холла, сопротивление $R_{H}$ пленок $\mathrm{Bi}_{2} \mathrm{Te}_{3}$ каждой ступени пропорционально не только обратной величине индекса уровня Ландау $n_{L}$, но и толщине образца или числу квинтетов $N_{q}$ - пятиатомных слоев толщиной около 1 нм, разделенных ван-дерваальсовой щелью. Холловское сопротивление, отнесенное к одному квинтету в единицах $h / e^{2}[7]$, соответствует обратным целочисленным значениям квантового эффекта Холла для трех уровней Ландау $\left(n_{L}=3,4,5\right)$ (рис. 2).

\section{3. Температурные зависимости гальваномагнитных эффектов}

Температурные зависимости магнетосопротивления и холловского сопротивления топологических изоляторов типа $\mathrm{Bi}_{2} \mathrm{Te}_{3}$ аналогичны зависимостям для топологических полуметаллов Дирака и Вейля [8] и содержат информацию о свойствах топологических поверхностных состояний в виде характерных плато при низких температурах (рис. 3-5). Температурные зависимости холловского сопротивления $R_{H}$ и магнетосопротивления $R_{x x}$ в магнитных полях $B=2.5,5$ и 10 Тл, нормированные к сопротивлению при $T=50$ и $100 \mathrm{~K}$, при низких температурах выходят на плато (рис. 3,4 ) вследствие топологического фазового перехода металл-изолятор. Отношения $R_{H} / R_{H}(T=100,50 \mathrm{~K})$ и $R_{x x} / R_{x x}(T=100,50 \mathrm{~K})$ возрастают с уменьшением температуры, т.е. поверхностный слой становится изолятором, в котором все электроны локализованы (рис. 3,4). При $B=0$ температурная зависимость сопротивления имеет явно выраженный металлический характер. Следует отметить, что магнетосопротивление на плато в халькогенидах висмута на несколько порядков меньше, чем в топологических полуметаллах, что коррелирует с более высоким значением отношения остаточного сопротивления $R_{R R}=R(T=290 \mathrm{~K}) / R(T=5 \mathrm{~K})$, характерным для металлов $[8,9]$.

Магнетосопротивление на плато нелинейно зависит от магнитного поля, и зависимости $R_{H} / R_{H}(T=100$, $50 \mathrm{~K})$ и $R_{x x} / R_{x x}(T=100,50 \mathrm{~K})$, построенные при

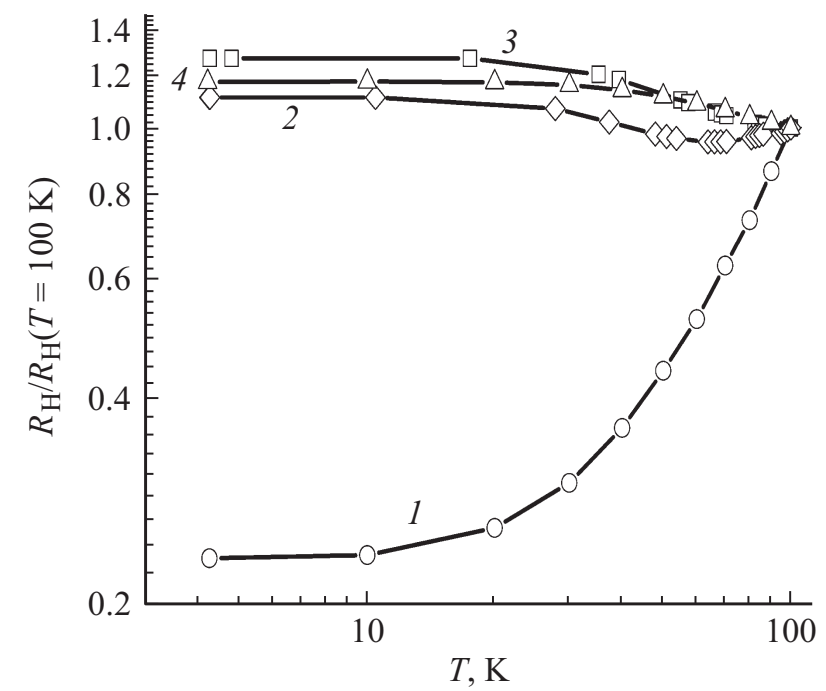

Рис. 3. Температурные зависимости холловского сопротивления $R_{H}$ в пленке $n-\mathrm{Bi}_{2} \mathrm{Te}_{3}$, нормированные к величине $R_{H}$ при $T=100 \mathrm{~K}$, в магнитных полях $B$, Тл: $1-0,2-2.5,3-5$, $4-10$.

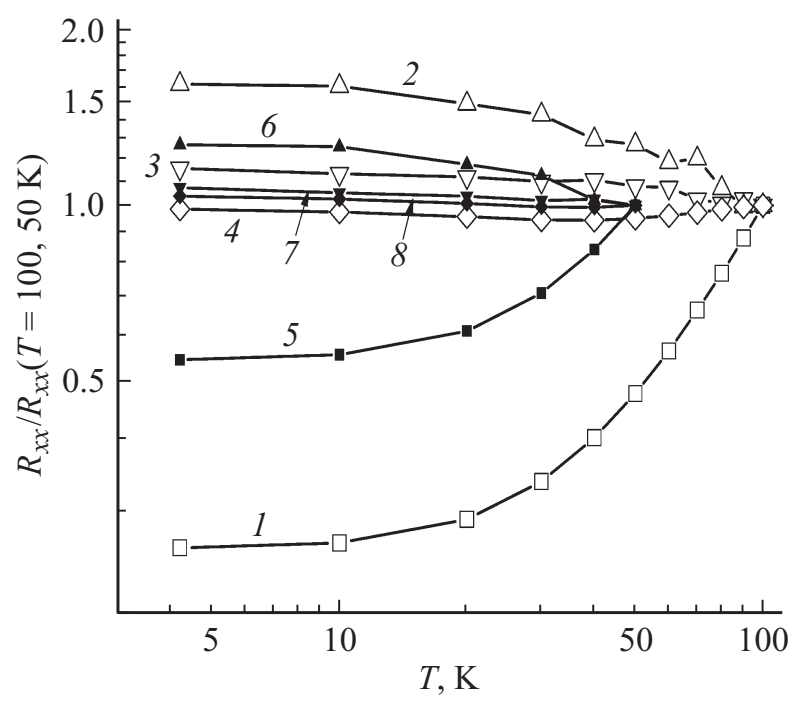

Рис. 4. Температурные зависимости магнетосопротивления $R_{x x}$ в пленке $n-\mathrm{Bi}_{2} \mathrm{Te}_{3}$, нормированные к величине $R_{x x}$ при $T=100(1-4)$ и $50 \mathrm{~K}(5-8)$ для тока $I$, направленного перпендикулярно $B$ и плоскости (0001). $B$,Тл: $1,5-0$; $2,6-2.5 ; 3,7-5 ; 4,8-10$. 


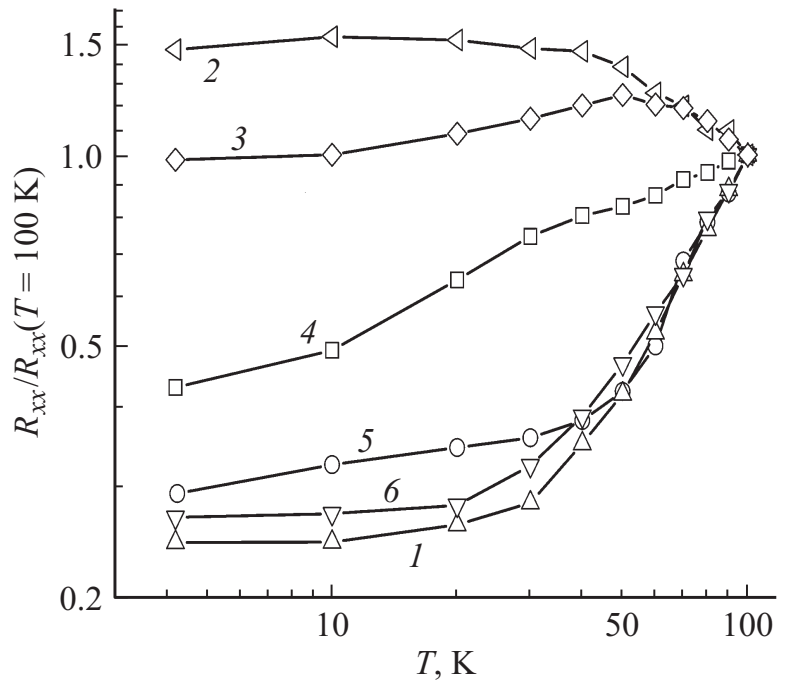

Рис. 5. Температурные зависимости магнетосопротивления $R_{x x}$ в пленке $n$ - $\mathrm{Bi}_{2} \mathrm{Te}_{3}$, нормированные к величине $R_{x x}$ при $T=100 \mathrm{~K}$, для тока $I$, направленного параллельно $B$ и плоскости $(0001)(2,3)$, и тока $I$, направленного перпендикулярно $B$ и параллельного плоскости (0001) (4-6). B, Тл: $1-0 ; 2,5-5$; $3,6-10 ; 4-2.5$.

$B=10$ Тл, располагаются ниже на рис. 3 и 4 , чем при меньших магнитных полях. Такую нелинейность можно объяснить зависимостью длины квантовой фазовой когерентности $l_{\phi}$ от магнитного поля [10]. Величина $l_{\phi}$ обычно много больше длины свободного пробега носителей заряда, что может приводить к появлению квантовых интерференционных эффектов, которые характеризуются слабой антилокализацией при уменьшении сопротивления поверхностного слоя в магнитном поле, или слабой локализацией, когда сопротивление возрастает [10]. Длина квантовой фазовой когерентности связана с процессами неупругого рассеяния электронов и ее можно оценить в виде $l_{\phi} \approx 700 \cdot T^{p / 2}$, где $p \approx 1$ в области малых магнитных полей при низких температурах [10]. По оценкам, в пленке $\mathrm{Bi}_{2} \mathrm{Te}_{3}$ величина $l_{\phi} \approx 350$ нм при $4.2 \mathrm{~K}$, а длина свободного пробега $l$, по нашим данным, равна около 60 нм [11]. При таких величинах $l_{\phi}$ квантовые эффекты, связанные с поверхностными состояниями дираковских фермионов, могут наблюдаться не только в сверхтонких образцах, поскольку, как было показано в [7] квантовый эффект Холла (рис. 2) зависит от количества квинтетов, т. е. от толщины образца. Обнаруженные топологические поверхностные состояния в достаточно толстых образцах до нескольких сотен нанометров при исследовании транспортных и оптических свойств, по-видимому, обусловлены влиянием большой длины квантовой фазовой когерентности $[2,7,11-13]$.

Плато на температурных зависимостях магнетосопротивления (рис. 4) существуют в области магнитных полей, при которых в пленках халькогенидов висмута и сурьмы были обнаружены осцилляции магнетосопротивления, связанные с топологическими поверхностными состояниями (рис. 1). Магнетосопротивление $R_{x x}$ (рис. 4) измерено, как и холловское сопротивление $R_{H}$ (рис. 3), при взаимно перпендикулярных направлениях векторов электрического тока $I$ и магнитного поля $B$, когда ток направлен параллельно кристаллографической плоскости (0001), что соответствует одному из поперечных компонентов тензора магнетосопротивления. При такой ориентации $I$ и $B$ осцилляции магнетосопротивления, связанные с топологическими поверхностными состояниями дираковских фермионов, имеют наибольшую амплитуду в пленках халькогенидов висмута и сурьмы [13]. Когда $I$ и $B$ взаимно параллельны и направлены параллельно плоскости (0001), наблюдаются продольные осцилляции магнетосопротивления, отношения $R_{x x} / R_{x x}(T=100 \mathrm{~K})$ возрастают, и плато на нормированных температурных зависимостях $R_{x x}$ также существуют (рис. 5, кривые 2,3). Для поперечного магнетосопротивления, измеренного при токе $I$, направленном перпендикулярно $B$, когда $I$ и $B$ параллельны плоскости (0001), не наблюдалось роста отношений $R_{H} / R_{H}(T=100 \mathrm{~K})$ в магнитном поле до 10 Тл (рис. 5, кривые 4,5). Низкотемпературное плато на нормированной зависимости $R_{x x} / R_{x x}(T=100 \mathrm{~K})$ возникало только при $B=10$ Тл, (рис. 5 , кривая 6 ). Как следует из угловых зависимостей осцилляций магнетосопротивления от магнитного поля, при такой ориентации $I$ и $B$ осцилляции не возникают [13]. Таким образом, рассмотренные нормированные температурные зависимости магнетосопротивления $R_{x x}$, измеренные при различных ориентациях тока и магнитного поля в пленках, могут быть использованы для обнаружения и анализа осцилляционных эффектов, связанных с влиянием топологических поверхностных состояний фермионов Дирака.

\section{4. Заключение}

Исследования топологических свойств поверхностных состояний дираковских фермионов в наноструктурированных пленках на основе теллурида висмута показали, что квантовый эффект Холла зависит от толщины образца и положения плато на холловском сопротивлении по отношению к одному квинтету обратно индексу Ландау. Относительные величины сопротивления плато на зависимостях магнетосопротивления и холловского сопротивления от температуры при $T$ ниже $15 \mathrm{~K}$ связаны с топологическим фазовым переходом металл-изолятор в магнитном поле.

Финансовая поддержка исследований частично получена в рамках проекта РФФИ № 16-08-00478.

\section{Список литературы}

[1] M.Z. Hasan, C.L. Kane. Rev. Mod. Phys., 82, 3045 (2010).

[2] Y.L. Chen, J.G. Analytis, J.H. Chu, Z.K. Liu, S.K. Mo, X.L. Qi, H.J. Zhang, H. Lu, X. Dai, Z. Fang, S.C. Zhang, I.R. Fisher, Z. Hussain, Z.X. Shen. Science., 325, 178 (2009).

[3] J. Lee, J. Koo, Y.M. Jhon, J.H. Lee. Opt. Express, 22, 6165 (2014). 
[4] Hong Qiao, Jian Yuan, Zaiquan Xu, Caiyun Chen, Shenghuang Lin, Yusheng Wang, Jingchao Song, Yan Liu, Qasim Khan, Hui Ying Hoh, Chun-Xu Pan, Shaojuan Li, Qiaoliang Bao. ACS Nano, 9, 1886 (2015).

[5] Y. Xu, Z. Gan, S.C. Zhang. Phys. Rev. Lett., 112, 226801 (2014).

[6] B. Seradjeh, J.E. Moore, M. Franz. Phys. Rev. Lett., 103, 066402 (2009).

[7] H. Cao, J. Tian, I. Miotkowski, T. Shen, J. Hu, S. Qiao, Y.P. Chen. Phys. Rev. Lett., 108, 216803 (2012).

[8] F.F. Taffty, Q.D. Gibson, N. Haldolaarachchige, R.J. Cava. Nature Physics, 2, 272 (2016).

[9] J.W.G. Bos, H.W. Zandbergen, M.H. Lee, N.P. Ong, R.J. Cava. Phys. Rev. B, 75, 195203 (2007).

[10] Hai-Zhou Lu, Shun-Qing Shen. Chinese Phys. B, 25, 117202 (2016).

[11] Л.Н. Лукьянова, Ю.А. Бойков, В.А. Данилов, О.А. Усов, М.П. Волков, В.А. Кутасов. ФТТ, 56, 907 (2014).

[12] K.M.F. Shahil, M.Z. Hossain, D. Teweldebrhan, A.A. Balandin. Appl. Phys. Lett., 95, 153103 (2010).

[13] Y.L. Chen, J.-H. Chu, J.G. Analytis, Z.K. Liu, K. Igarashi, H.-H. Kuo, X.L. Qi, S.K. Mo, R.G. Moore, D.H. Lu, M. Hashimoto, T. Sasagawa, S.C. Zhang, I.R. Fisher, Z. Hussain, Z.X. Shen. Science, 325, 178 (2010).

Редактор Г.А. Оганесян

\title{
Transport properties of heteroepitaxial films based on bismuth telluride in strong magnetic fields
}

\author{
V.A. Danilov, M.P. Volkov \\ loffe Institute, \\ Russian Academy of Sciences, \\ 194021 St. Petersburg, Russia
}

L.N. Lukyanova, Yu.A. Boikov, O.A. Usov,

Abstract Temperature and field dependencies of galvanomagnetic properties of $n-\mathrm{Bi}_{2} \mathrm{Te}_{3}$ heteroepitaxial films were studied in magnetic fields up to $14 \mathrm{Tl}$ at low temperatures. It is shown that resistance steps on the magnetic field dependence of the quantum Hall effect and low temperature magnetoresistance plateau in the films are related to topological surface states of Dirac fermions. 